\title{
A Review of the Research on the Mechanical Model of Vehicle Tires
}

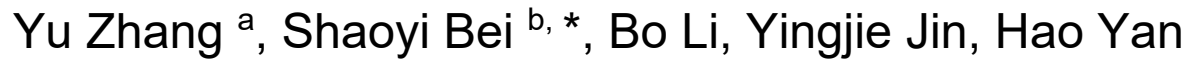

Jiangsu University of Technology, College of Automotive and Transportation Engineering, China.

a 1916571064@qq.com, *,b bsy1968@126.com

Keywords: Tire model; Magic Formula; UniTire; Neural network.

\begin{abstract}
Tire as the only ground part of the vehicle, which is transmitted between the vehicle and the ground force of the media, with supporting the weight of the vehicle, vehicle running in different ground buffers, provide sufficient adhesion for driving and braking, provide adequate steering and direction stability. The mechanical characteristics of the tire are directly related to the driving stability and steering performance of the vehicle, which is the basis of the vehicle dynamics. The further development of automobile dynamics and its control technology depends on the precise tire mechanics model technology. In this paper, the research of tire mechanics model is reviewed from the point of view of modeling method, including the physical model, the empirical model, Semi empirical model and the adaptive model. The advantages and disadvantages of the typical models and the application fields are also pointed out. Finally, future study of the problems and the development trend of automobile dynamic tire model is summarized.
\end{abstract}

\section{Introduction}

Tires are an important part of a car. The structural parameters and mechanical properties of the tire determine the main driving performance of the car. In addition to air force and gravity, almost all other forces and moments that affect vehicle motion are generated by the interaction of rolling tires with the ground. For the driving vehicle, the vertical force, longitudinal force, lateral force and moments back to the tires are very important for studying its dynamics, including control stability, ride comfort and braking safety. Therefore, it is necessary to establish an accurate model of tire mechanics to simulate the vehicle movement under the actual driving condition and then to analyze and simulate the vehicle [1]. However, the force of the tire is very complex during the driving process. It varies according to the nature of the road, the speed of the vehicle, the vertical load, the temperature at which friction occurs and the form of the tire. And the tire has the complexity of structure and the nonlinearity of mechanical properties. Therefore, establishing a tire model that matches the precision of the vehicle model has always been the focus and difficulty for domestic and foreign scholars to discuss, and it is also one of the problems to be solved urgently in the automobile industry.

\section{Research Status of Tire Model}

Since the development of tire dynamics model, from the study of steady-state characteristics to the analysis of Non-steady-state characteristics, until the discussion of adaptive tire models in recent years, we can say that the tire model has been quite rich. Tire models can be divided into four categories: physical model, empirical model, semi-empirical model and adaptive model [2].

After analyzing the mechanical characteristics of tires, the tire physical model is approximated as four parts: rim, carcass, belt and tread by reasonable physical simplification. Such simplification is of great significance for analyzing the mechanical properties of tires and structural parameters. However, its general form is more complex, the model accuracy and computational efficiency are lower. Such as string model, beam model, brush model [3], finite element model [4], SWIFT model [5] and FTire model [6]. The tire experience model is a tire model that shows the mechanical properties between the tire and the road surface based on recursive analysis of a large amount of test data. It is through testing an existing tire to make the parameters obtained from the analysis curve match the parameters obtained in the actual test, so as to achieve the purpose of building the tire model. The accuracy of the model is high, and its prediction ability is worse than that of the physical 
model, such as the Magic Formula model [7]. Tire semi-empirical model is based on the tire physical model by meeting certain boundary conditions to establish a simple and highly accurate tire empirical model and it is easy to be applied in automobile dynamics simulation. Such as Guo Konghui's UniTire model [8]. The adaptive tire model is based on theoretical and experimental data. It establishes an intelligent model with certain adaptability to the external environment by simulating some structures and functions of organisms and aiming at various input parameters. This model has high modeling efficiency and considerable accuracy, such as neural network tire model [9].

\section{Research on Modeling Method of Tire Mechanical Model}

Tire mechanical model from the use can be divided into: stability model, comfort model and fatigue load model; from the performance can be divided into: steady-state model, transient model and dynamic model; from the modeling methods can be divided into four categories: physical model, empirical model, semi-empirical model and adaptive model [2, 10]. This paper classified the tire mechanics model according to the tire modeling method.

\subsection{Tire Physics Model}

\section{String Model}

The string model was first introduced by Von Schlieppe of Germany to describe the mechanical behavior of rolling tires. He simplified the tire carcass to the stretched chord. The string had no mass so that it was able to bear the tension and lateral resilience. The elastic restoring force represented the spring stiffness of the tire side. The string model gives the analytical transfer function between the yawing force / back moment and the tire steering angle. This is particularly important for the study of steering wheel shimming. Therefore, it is widely used in the research of aircraft landing gear shimmy and steering wheel shimmy. So far, some scholars are still working on the improvement of the string model.

Beam Model

A string cannot bear bending, so Fromm and Fiala have improved the string model. They assumed the carcass as an infinite length beam on an elastic basis, the tread assumes the same assumption as the brush model and extroversion and relaxation length are not considered. When the internal dip is not considered as the main factor and the longitudinal and transverse slip are treated separately, a reasonable result can be obtained for simple maneuverability analysis. The Fiala model also gives the analytical solution of the lateral force and the back moment. Compared with the string model, it can reflect the deformation physics of the tire [10].

\section{Brush Model}

The brush model is mainly based on the assumptions of elastic tread and rigid carcass. It is simulated by a three-dimensional elastic brush unit. The hypothesis mainly includes: (1) The elasticity of the tire is completely concentrated on the deformable brush of the tread, and the tire body is regarded as a rigid body. (2) The length of the grounding imprinting is fixed and the amount is known. (3) The distribution of vertical ground pressure is known. (4) Friction between the tire and the pavement is Coulomb friction. The most important feature of this model is that the computing speed is fast and the required parameters are less. It can be used to analyze the relationship between lateral force and side angle, longitudinal slip and longitudinal force. It is commonly used in the control and stability analysis. Many of the stable tire models are derived on this basis, such as the UniTire tire model proposed by Academician Guo Konghui [11].

Finite Element Model

The finite element model is used to describe the geometric characteristics of the tire and the material properties of each layer in detail [4]. The basic idea of the finite element method is to discrete the continuum into a unit and units connected by nodes. The element characteristics are determined by the interpolation function. Add the unit characteristics to get the overall tire characteristics and then it is solved by a computer. Finally, the total matrix is formed and get the total structural response. The greatest advantage is that it can simulate any complex structure and load. 
Because finite element analysis can determine the response at each point inside the tire and can predict the performance of the tire, so it has been widely used. At present, this technology has been used to analyze the inflatable shape and stress, load displacement curve, the ground area and shape, the earth pressure, the total force and moment, and the natural frequency. At the same time, the key technologies and difficulties faced by the finite element analysis of tires are: tire structure and configuration of the precise simulation, reliable tire material properties, analysis of large deformation and contact problems [12-13].

SWIFT Model

The full name of the SWIFT model is a short wave medium frequency tire model. The model was jointly developed by the Delft industry university in Holland and TNO [5, 14]. It is a rigid ring model. On the basis of the ring model, only two order modes of the 0 order and 1 order dislocation of the tire are considered. At this point, the tire is only rigid body motion without deformation [15]. The requirement is satisfied when only the medium and low frequency characteristics of the tire are concerned. Because there is no need to calculate the deformation of the carcass, so it can be used for the development of active suspension and ABS in the loop simulation.

The SWIFT model simplifies the belt to a rigid ring with 6 degrees of freedom. It connects the ground mass with 3 degrees of freedom between the rigid ring and the road surface. The improvement of the model compared with the simple physical model lies in the separation of the modeling work between the carcass and the grounding area. In addition, considering the inertia of the belt, so that it is treated as a rigid ring. In order to ensure the correct static stiffness of the tire in all directions, a flexible unit is introduced to characterize the residual stiffness. The modeling of the tread still uses the assumption of a classic brush model. In the simulation of the tires rolling across different surfaces, it replaces the actual pavement with an effective pavement using three values of pavement height, slope and effective roll radius that reflects tire containment characteristics. The six force characteristics of the model in the nonlinear region are described by the magic formula model [10].

The structure of the SWIFT model has the following four characteristics [16]:

The carcass part adopts the rigid ring theory, the tread part uses the magic formula. It uses a modeling method that separates the carcass from the ground area. The magic formula is used to calculate the lateral force and the back moment, the longitudinal force and the vertical force are calculated by the rigid ring theory.

The belt quality and moment of inertia are considered. In the high frequency range, the belt is assumed to be a rigid ring. The residual stiffness is introduced between the ground mass and the rigid ring. Vertical stiffness, longitudinal stiffness, lateral stiffness is equal to the static stiffness of the tire in all directions.

The length and width of the grounding imprinting are considered.

The pavement characteristics are described by effective pavement height, pavement slope and effective rolling radius of the tire with an inclusive characteristic. Realize the tire in any 3D uneven road simulation to ensure the tire dynamic slippage and vibration simulation conditions.

Ftire Model

FTire was developed by a team led by Michael Gipser of the University of Esslingen in Germany [6]. It is a complete three-dimensional nonlinear simulation model in the plane and outside the plane. The model describes the belt through elastic ring (radial, tangential and lateral) structures with a certain bending stiffness and tensile stiffness. The implicit integration core method is used to calculate the shape of the belt. The model can be used to simulate belt dynamics, ground pressure distribution, roll hysteresis, sidewall contact, large camber and tire misuse [17].

The core idea of FTire model modeling is to replace the tire steel ribbon bundle with 80 to 200 concentrated beam nodes, and the nodes are connected by spring damping. These nodes are subjected to 3 combinations of deformation: moving in 3 directions, rotating around the circumference and bend laterally. Belt nodes are connected to the rim through four different types of elastic elements to simulate the radial characteristics of the tire. To ensure the flexibility of belt node, there are threeway torsional deformations between adjacent nodes or nodes and rims to simulate side effects. In the tire ground area, according to the needs of the actual problems, inserting an appropriate number of 
mass free contact elements into the adjacent belt nodes. Then a special program is used to quickly determine whether contact units are in contact with the ground. Finally, the pressure distribution in the ground area is obtained [10].

\subsection{Tire Experience Model}

The Magic Formula tire empirical model proposed by Pacejka is widely used in the field of vehicle dynamics research [7]. The Magic Formula tire model fits the tire test data using a combination of trigonometric functions. It can fully express the relationship between the force and torque of a tire under different driving conditions by using the same basic formula. The core formula of the Magic Formula tire model is shown as follows [18]:

$$
\begin{gathered}
\mathrm{Y}(x)=\mathrm{D} \sin \{\operatorname{Carctan}[B x-E(B x-\arctan (B x))]\} \\
\mathrm{Y}(X)=Y(x)+S_{V} \\
\mathrm{x}=\mathrm{X}+S_{H}
\end{gathered}
$$

In the formula: $\mathrm{Y}(X)$-Lateral force of output variable $F_{y}$ or longitudinal force $F_{x} . \mathrm{X}$-Side angle of input variable $\alpha$ or Longitudinal slip rate K. B-Stiffness factor. C-Shape factor. D-Peak factor. ECurvature factor. $S_{H}$ And $S_{V}$ - Horizontal and vertical deviation (Considering the original deviation of the tire with the initial side angle).

The process of generating basic curve of the magic formula and the meaning of each parameter are shown in Figure 1. Magic formula tire empirical model $\mathrm{Y}(x)$ produces a maximum of typical curves that pass through the origin and then converges to a horizontal asymptote. If the values of the coefficients B, C, D, and E have been given, then the curve will show an asymmetric shape about the origin. Deviation $S_{V}$ and $S_{H}$ may be caused by the side force or rolling resistance, it causes the curve $F_{x}$ and $F_{y}$ not to pass through the origin. The curve produced by this formula closely matches the actually measured lateral force $F_{y}$ and longitudinal force $F_{x}$ curve [19].

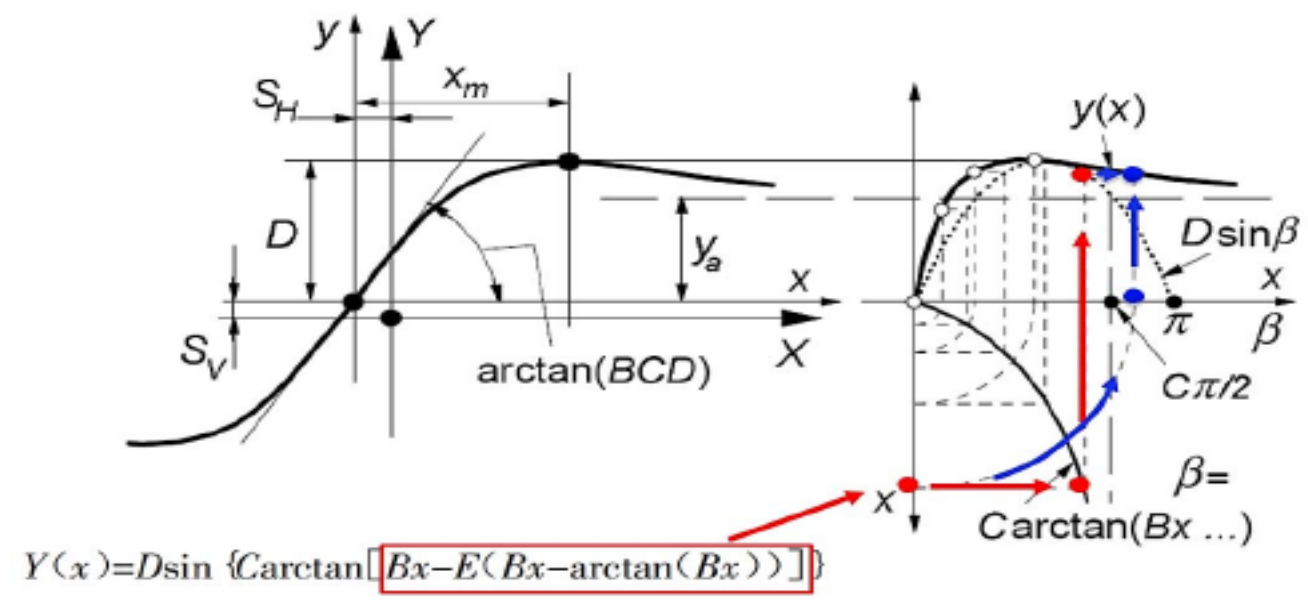

Figure 1. Curve Generation Process and the Meaning of Each Parameter

The formula for calculating the tire trailing distance is shown as follows [18]:

$$
\begin{gathered}
\mathrm{t}\left(\alpha_{t}\right)=D_{t} \cos \left[C_{t} \arctan \left\{B_{t} \alpha_{t}-E_{t}\left[B_{t} \alpha_{t}-\arctan \left(B_{t} \alpha_{t}\right)\right]\right\}\right] \cos (\alpha) \\
\alpha_{t}=\alpha+S_{H t} \\
M_{Z}=-t \cdot F_{y 0}+M_{z r}
\end{gathered}
$$

At this time, $D_{t}$ is still the peak parameter. $C_{t}$ Is still the shape parameter. Essentially, due to the cosine curve does not pass zero, so the role of $B_{t}$ is mainly to affect the curvature of the peak. $E_{t}$ Mainly affects the shape of the curve at large side declination angle and it determines the position of the intersection of curve and transverse axis. The process of generating the trailing distance curve is shown in Figure 2. 


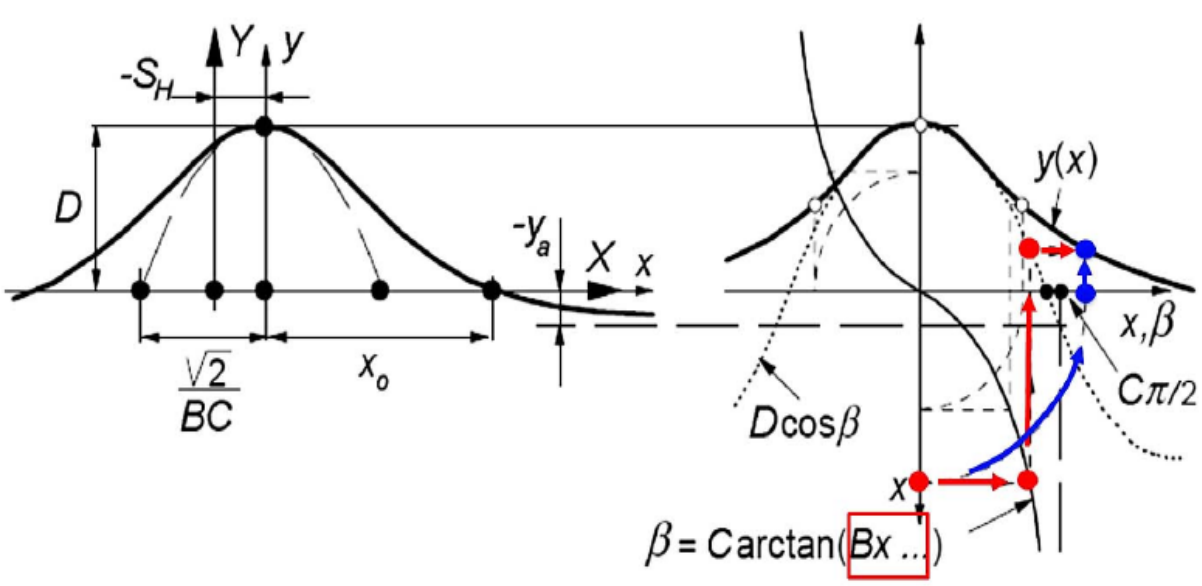

Figure 2. Trailing Distance Curve Generation Process

In general, the magic formula model has a higher fitting accuracy for steady six-component force data. It can be used for vehicle driving test support and Control System Design Analysis. But its disadvantage is that it's fitting parameters too much. Each of the six parameters $B_{t}, C_{t}, D_{t}, E_{t}, S_{v t}$, $S_{H t}$ described above requires a large number of sub-parameters to control (The number of sub parameters depends on the model version and the simulation requirements). Therefore, the cost of fitting the parameters of the magic formula model is very high. In addition, this method cannot give the mechanism and action of the six-component force from the structural level, and then puts forward the design improvement opinion [19].

\subsection{Semi Empirical Model}

Guo Konghui proposed and built the UniTire tire model which is based on Fiala's simplified tire physics model. The model studies the influence of the deformation characteristics of the carcass on the tire lateral mechanical properties from the general principle. And it is through experiments to identify the deformation parameters of the carcass. It is not only able to express the high precision of the tire mechanical properties in various working conditions, but also it has good extrapolation and predictive ability. It can accurately predict the tire characteristics at different conditions, different pavement and different speeds. In the whole modeling process, we use the physical model as the basis, and use the dimensionless expression form. The model insists on the basic idea of separating the friction coefficient and has uniform definition of slip rate $[8,20]$. In order to unify the tire lateral and longitudinal slip rate, the coordinate system of the UniTire tire model is based on the imprinted coordinate system. The coordinate system is also called the CP coordinate system [21-22], as shown in Figure 3.

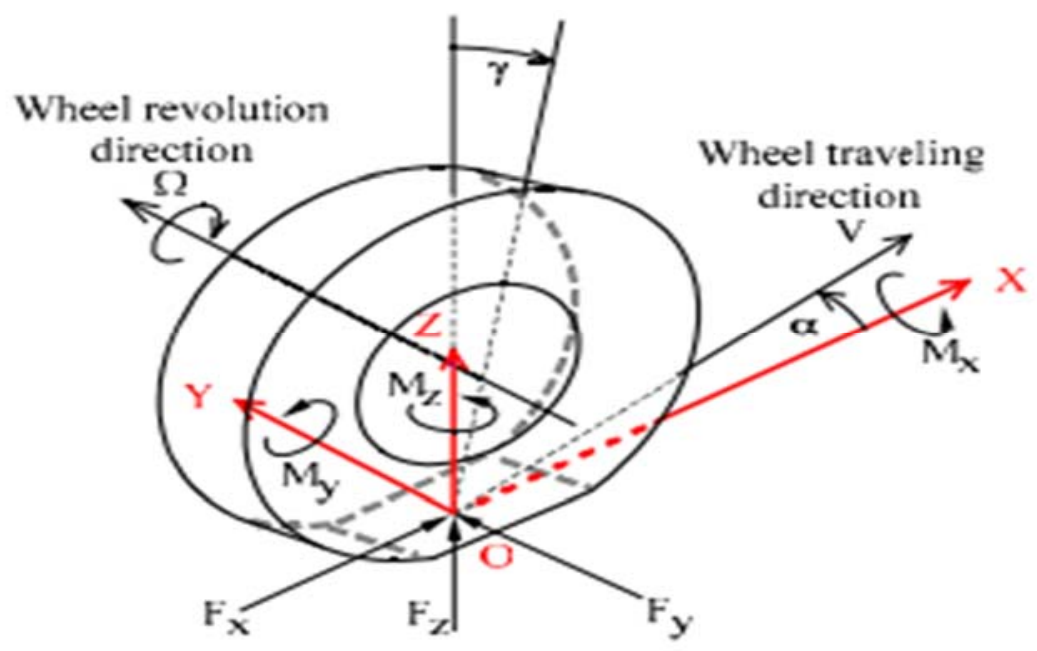

Figure 3. Unitire Tire Imprinted Coordinate System 
The core expression of the UniTire semi empirical tire model proposed by Professor Guo Konghui is shown as follows. It is used to describe the dimensionless total shear force $\bar{F}$ and the righting arm $D_{x}$ of the tire under the combined working condition of longitudinal slip and slide deviation [23-24].

$$
\begin{gathered}
\bar{F}=1-\exp \left[-\emptyset-E \emptyset^{2}-\left(E^{2}+1 / 12\right) \emptyset^{3}\right] \\
D_{x}=\left(D_{x 0}+D_{e}\right) \exp \left(-D_{1} \varnothing-D_{2} \emptyset^{2}\right)-D_{e}
\end{gathered}
$$

In the formula, E-Curvature factor of the total shear force curve. It can adjust the bending degree of the curve in the transition zone. $D_{x 0}$-Initial righting arm. $D_{e}$-Final value righting arm. $D_{1}$ And $D_{2^{-}}$ The curvature factor of the righting arm curve. $\varnothing$-Relative total slip rate.

Define longitudinal slip rate $S_{x}$, Lateral slip rate $S_{y}$, Dimensionless relative longitudinal, lateral and total slip rates $\emptyset_{x}, \emptyset_{y}$ and $\emptyset$.

$$
\begin{array}{ll}
S_{x}=\frac{-v_{s x}}{\Omega R_{e}}=\frac{\Omega R_{e}-v_{x}}{\Omega R_{e}} & S_{x} \in(-\infty,+\infty) \\
S_{y}=\frac{-v_{s y}}{\Omega R_{e}}=\frac{-v_{y}}{\Omega R_{e}} & S_{x} \in(-\infty,+\infty) \\
\emptyset_{x}=\frac{K_{x} S_{x}}{\mu_{x} F_{z}} \quad \emptyset_{y}=\frac{K_{y} S_{y}}{\mu_{y} F_{z}} \quad \emptyset=\sqrt{\emptyset_{x}^{2}+\emptyset_{y}^{2}}
\end{array}
$$

In the formula, $\Omega$-Tire rolling angular velocity. $v_{x}, v_{y}$-The component of the velocity of wheel in longitudinal and lateral directions. $R_{e}$-Effective rolling radius. $K_{x}, K_{y}$-The longitudinal and lateral stiffness of the tire. $\mu_{x}, \mu_{y}$-Friction coefficient of longitudinal and lateral in contact imprinting. $F_{Z^{-}}$ Tire vertical load.

When the longitudinal and lateral stiffness are different, especially when the slip is relatively large, the model needs to be modified. So, make the following definition:

$$
\varphi_{n}=\sqrt{\left(\lambda \varphi_{x}\right)^{2}+\varphi_{y}^{2}}
$$

Thus, the longitudinal force, lateral force and positive torque of the tire are expressed as follows.

$$
\begin{gathered}
F_{x}=\bar{F} \frac{\varphi_{x}}{\varphi_{n}} \mu_{x} F_{z} \\
F_{y}=\bar{F} \frac{\varphi_{y}}{\varphi_{n}} \mu_{y} F_{z} \\
M_{z}=F_{y} \cdot\left(D_{x}+X_{C}\right)-F_{x} \cdot Y_{c}
\end{gathered}
$$

In the formula, $X_{C}, Y_{C^{-}}$The offset of the actual imprinting center relative to the theoretical imprinting center caused by the longitudinal and lateral forces of the tire.

\subsection{Adaptive Model}

The tire adaptive model which develop more mature at present is the neural network tire model. It is based on theoretical analysis and experimental data. The artificial neural network principle is used to establish a tire model that allows multiple input and multiple output, and it also has a certain adaptive ability. Due to the model has strong prediction ability, parallel ability and nonlinear approximation ability under the action of nonlinear force, so it has a good prospect in the field of tire dynamics modeling and simulation $[1,25]$.

The neural network system is a highly complex nonlinear dynamic system. The system consists of a large number of neuron nodes. Its structure and characteristics are determined by the characteristics of neurons and the connection between them. Neurons are the basic processing units in the neural network. Although the structure of each neuron is relatively simple and its function is limited, but a large number of neurons are connected to a network in a certain way and to adjust the strength of the connections between neurons, so that it makes the system very powerful. In neural networks, the process of information processing or the change of storage knowledge is accomplished by modifying the mode of connection between neurons. The process of modification is called the learning or training of the neural network. The learning process of neural networks is the process of continuously modifying the connection strength between neurons [25-26].

There are three neurons in the input layer, and their inputs include Vertical load of tire, Camber Angle and Side-slip Angle. There is a neuron in the output layer, it can output lateral force or positive torque of the tire. The number of neurons in the hidden layer is determined by the number of training samples. Under the learning conditions of two kinds of camber angle, three kinds of vertical loads 
and nine kinds of side-slip angle, it can form a network structure of $2 \times 3 \times 9$ and correspond to 54 teacher values. After forming the neural network structure, it uses BP algorithm and the corresponding learning sample and teacher value are used for training. At the end of the calculation, the learning phase is over, it can get the network weight matrix and constitute the lateral force, back positive force matrix neural network model $[9,26]$.

\section{Conclusion and Prospect}

So far, research on the steady-state characteristics of tires has been quite mature. Many scholars have given a complete description of the tire model, it can meet the requirements of vehicle system dynamics research and simulation. In contrast, although significant progress has been made in the study of the non- steady- state characteristics of the tires, but most of the theoretical research is limited to a small range of motion. There is no longitudinal and transverse slide in the whole imprinting area. It does not consider the quality and inertia of the tires or only emphasize the dynamic hysteresis effect of lateral force and positive torque. In the field of experimental research, the test equipment and measuring sensors are still unable to meet the tire dynamic characteristics of high frequency and large range. Therefore, it is the main research direction of tire modeling to establish the nonlinear model of high speed, high frequency, large range and transient condition based on neural network. How to effectively consider the tire working conditions and the impact of the tire material, structure, size and pattern and other factors will be the difficulty of the modeling.

\section{Acknowledgements}

This project is supported by the National Natural Science Foundation of China (Grant No. 51705220), the Jiangsu Province Higher Education Natural Science Research Project (17KJD580001) and the Jiangsu Provincial Higher Education Natural Science Research Major Project (17KJA580003) and Foundation for Jiangsu Province "333 Project" Training Funded Project (BRA2015365), Changzhou Science and Technology Support Project (Industry) (CE20150084).

This project is supported by the National Natural Science Foundation of China (Grant No. 51705220), the Jiangsu Province Higher Education Natural Science Research Project (17KJD580001) and the Jiangsu Provincial Higher Education Natural Science Research Major Project (17KJA580003) and Foundation for Jiangsu Province "333 Project" Training Funded Project (BRA2015365).

\section{References}

[1]. Wang Yupeng, Zhao Longqing. Typical tire model and its development trend [J]. Agricultural equipment and vehicle engineering, 2006 (12): 3-5.

[2]. Wang Heyi, Gu Zhengqi. Research status and development analysis of automobile tire model [J]. Rubber industry, 2005, 52:58-63.

[3]. Guo K H, Ren L.A unified semi-empirical tire model with higher accuracy and less parameters. SAE Technical Paper Series, 1999-01-0785.

[4]. Noor A K, Tanner J A. Computational Modeling of Tires[C]. NASA-CP-3306, 1995.

[5]. Besselink I J M, Pacejka H B, Schmeitz A, et al. The MF-Swift tyre model: Extending the Magic Formula with rigid ring dynamics and an enveloping model [J]. Review of Automotive Engineering.2005, 26(2):245-252.

[6]. Gipser M. F Tire-the tire simulation model for all applications related to vehicle dynamics [J]. Vehi Syst Dyn, 2002, 45:139-151.

[7]. Pacejka H B. Tyre and Vehicle Dynamics [M]. Vol.93-134: Butterworth-Heinemann.2002. 
[8]. Guo Konghui. The principle of automobile manipulation dynamics [M]. Nanjing: Jiangsu Science and Technology Press, 2011.

[9]. Zhang Jianwei. Research on the mechanical model of automobile tires [D]. Hubei: Wuhan University of Technology, 2006.

[10]. Wei Yintao, Feng Xijin, Feng Qizhang, Liu Yuan, He Yuan. Research progress on dynamic model of tires [J]. Journal of automotive safety and energy conservation, 2014, 5 (4): 311-323.

[11]. Liu Qing, Guo Konghui. Analysis of tire brush model - steady-state lateral brush model [J]. Journal of agricultural machinery, 2000, 31 (1) 19-22.

[12]. FU Jingjing. Research on UniTire modeling method based on tire design parameters [D]. Changchun: Jilin University, 2013.

[13]. Wei Yintao, Du Xingwen. Nonlinear finite element analysis of automobile tire structure [J]. Automotive engineering, 1998, 20 (5): 272-278.

[14]. Schmeitz A J C, Versteden W D. Structure and Parameterization of MF-Swift, a Magic Formula-based Rigid Ring Tire Model [J]. Tire Science \& Technology, 2009, 37(37):142-164.

[15]. Besselink I. Appendix 3-MF-Tire/MF-Swift Parameters and Estimation Methods [J]. Tire \& Vehicle Dynamics, 2012:613-625.

[16]. Yu Fan, Lin Yi. Automotive system dynamics [M]. Machinery Industry Press, 2005.

[17]. Fei Ruiping. Simulation analysis and experimental research on FTire tire model [D]. Changchun: Jilin University, 2011.

[18]. Yuan Zhongcheng, Lu Dang, Guo Konghui. Comparison of UniTire and Magic Formula steady state model [J]. Design, Calculation and Research, 2006 (2): 7-9.

[19]. Zhang Haitao. Comparison of SWIFT and FTire tire model [D]. Changchun: Jilin University, 2016.

[20]. Guo Konghui. UniTire Tire Model [J]. Journal of Mechanical Engineering, 2016, 52 (12): 9099.

[21]. Xu Nan. Study on the steady state model of tire under compound condition [D]. Changchun: Jilin University, 2012.

[22]. Jin Lingge. The connection of the UniTire model and the dynamics software [D]. Changchun: Jilin University, 2006.

[23]. Guo Konghui, Jin Lingge, Lu Dang. The application of UniTire model in vehicle dynamics simulation [J]. Journal of Jilin University: Engineering Edition, 2009, 39 (2): 241-245.

[24]. Yuan Zhongcheng. Study on the steady state model of tires [D]. Changchun: Jilin University, 2006.

[25]. Cui Shengmin, Wang Fei. Lateral characteristics model based on artificial neural network [J]. Tire Industry, 2000, 20 (1): 11-14.

[26]. Ren Weiqun, Huang Miaohua, Jin Guodong, Zhang Yunqing, Fu Yongzhi. Manipulation stability simulation based on neural network tire model [J]. Computer Simulation, 2000, 17 (2): 46-49. 